\title{
Implementing Clinical Practice Guidelines for Chronic Obstructive Pulmonary Disease in an EHR System
}

\author{
Marisa Walker, Weiwei Ge, Judy W. Gichoya and Saptarshi Purkayastha
}

\begin{abstract}
The use of clinical practice guidelines to improve quality of care has been a vividly discussed topic. Clinical practice guidelines (CPG) aim to improve the health of patients by guiding individual care in clinical settings. CPGs bring potential benefits for patients by improving clinical decision making, improving efficiency and enhancing patient care, while essentially optimizing financial value. Chronic conditions like heart disease, stroke, and chronic obstructive pulmonary disease (COPD), plague the US healthcare system causing several million dollars in healthcare related cost. This paper demonstrates the development of a CPG into an open- source EHR system to effectively manage COPD patients. The CPG is incorporated using the open web app standard, which allows it to be used with any web browser based EHR system, once data from the EHR system can be fed into the app. As a result, the CPG helps create a more effective and efficient decision-making process.
\end{abstract}

\section{INTRODUCTION}

Clinical practice guidelines (CPGs) are designed to create a protocol of care for patients that makes treating patients with certain disease types simpler [1]. The effective use of CPG has been shown to reduce hospitalization, emergency department visits, and outpatient visits as shown in the Cloutier study [2]. The use of asthma guidelines in this study also showed an increased adherence by providers that additionally effected patient outcomes [2]. In fact, in 2010 the projected expenditure in managing COPD care in the US was an estimated \$50 billion in direct and indirect costs [3]. For this reason, it is vital that electronic health records (EHR) systems and clinical practice guidelines should be integrated to improve efficiency and patient safety.

CPGs are evidence based practices that are usually released in medical literature and clinicians have to keep these CPGs in their minds while providing care [4]. With a large number of patients under care for chronic conditions like COPD and visits scheduled as far as 3-6 months, it is very hard for clinicians to remember all the case details about a patient [5]. Thus, clinicians spend a lot of time reviewing patient history in the EHR systems, and since CPGs are often updated due to new evidence, the integration of the patient history information with CPGs becomes critical for patient safety and appropriate patient care.

The 2014 Veterans Affairs (VA)/Department of Defense (DoD) Management of Chronic Obstructive Pulmonary Disease Clinical Practice Guidelines [6] were chosen for incorporation into the EHR system. The VA/DOD guidelines were updated in 2013. These guidelines, as written in the

M. Walker, W. Ge and S. Purkayastha are with the Department of BioHealth Informatics, Indiana University Purdue University Indianapolis, Indianapolis, IN 46202 USA. (e-mail: saptpurk@iupui.edu). qualifying statement, were developed to assist providers in decision making for the management of COPD. They are not considered a standard of care and are not specific to the VA/DOD patients such as military personnel or veterans. The guidelines can be used in a multitude of settings provided in the algorithms and guidelines. The list includes several recommendations on the diagnosis and treatment of these patients. The clinical guidelines also include algorithms for providers to follow as shown in Figure 1 below. The algorithm included in Figure 1 displays the decision-making process of a newly suspected or confirmed diagnosed COPD patient. After completing the initial clinical assessments of the patient, the provider is then led to deciding whether the patient is acute or chronic which leads to another algorithm. The algorithm essentially ends with the provider providing either follow up care, initiating pharmacotherapy, oxygen assessment, or management of the condition using other therapies.

In this paper, we present the development of the VA/DoD CPG on managing COPD, as an open web app in an electronic health record system. We describe the novel single page userinterface that simplifies scheduling, prioritization, treatment ordering for all patients under care of a provider user.

\section{METHOD}

The project was initially worked on by a nurse, who is also a graduate student in the Health Informatics program at Indiana University Purdue University Indianapolis. A literature search was completed to look at the various available CPGs for COPD management in PubMed, Scopus and Google Scholar. Based on the search result, only guidelines that were used in research studies or implemented in practice were selected for further analysis. CPGs published before 2002 were discarded, since COPD management has improved in the last 15 years. 14 different CPGs for COPD were finally assembled and analyzed for differences and similarities. On comparison of the 14 , we discovered that only 4 of these were different in the guidelines. Since the main subject of the paper is development of the selected CPG into the EHR system, we do not present the detailed analysis of the CPGs. But the 4 CPGs that need to be mentioned - The COPD CPG of (i) AMDA - The Society for Post-Acute and Long-Term Care Medicine; (ii) Academy of Medicine of Malaysia; (iii) The Global Initiative for Chronic Obstructive Lung Disease (GOLD); (iv) VA/DoD CPG. These guidelines also identify the factors needed to diagnosis of a patient with COPD. The recommendations included in these guidelines account for the specific parameters needed to diagnose a patient as well as treat. One of the parameters involved in diagnosing a patient

J. W. Gichoya is with the Indiana University School of Medicine, Indianapolis, IN 46202 USA. 
with COPD pertain to the vital signs that are captured during examination as well as confirmation through testing.

Using the guidelines, it was found that the FEV1, partial pressure of oxygen in the blood (PaO2), and FEV1/FVC ratio were the most important factors in the diagnosing and treatment COPD. Based on the observations that are made, the patient is categorized into five categories of severity and pharmaceutical intervention is recommended based on the categorization. The guidelines as well as the algorithms serve as a means to provide a quick reference and recommendation on treatment options.

We selected the OpenMRS EHR system, since it is used by the Health Informatics program at IUPUI, by importing deidentified data from 7 CHCs in Indiana that were part of a PCORI grant [7]. We also use OpenMRS by integrating it with a single sign-on system, such that data can be shared in a secure way even by using Basic Authentication over HTTPS [8]. These two situations somewhat defaulted the use of OpenMRS, as a choice of the EHR system to implement this COPD CPG. To be able to capture the clinical observations, we needed to create the concepts in the OpenMRS concept dictionary. The concept dictionary defines all medical concepts and descriptions contained in OpenMRS. These medical concepts were also linked to other medical commonly used terminologies such as LOINC and SNOMED. The concept for COPD was already available in the default concept dictionary with the name chronic obstructive pulmonary disease and the assigned UUID was used to retrieve data related to the concepts between the app and the EHR system.

The novelty in the development of the app is the use of the Open Web App (OWA) standard [9, p. 25]. The OWA standard is defined by W3C and is a standard packaging format of HTML, CSS, JavaScript and other native web browser technologies that can be used to build applications that can run across browsers, mobile devices [10] and even devices such as televisions and wearable devices. The OWA standard has also found some application in the health-space such as the TCGA toolbox [11], although the SMART application platform has got much more traction in the health space [12]. The SMART app platform is used as an enabler for substitutable applications for EHR systems. OpenMRS had supported SMART apps in a rudimentary way for quite some time, but has fairly recently adopted OWA packaging for applications, thus using REST API for data exchange between modular parts of the system, such as OWA apps and the core EHR system platform. We used the Yeoman (www.yeoman.io) script that allows creating a template for a package OWA application [13], which runs on the Node.js runtime environment. Node.js is the basic framework for building the web application but the libraries needed to build the OWA package were provided by the Yoeman script. The Node Package Manager (npm) allows installation of the yeoman script, as well as setting up the environment to deploy the OWA application to OpenMRS for testing purpose.

After creating the OWA template files were created, we needed to setup a common repository through which we could collaborate in the development of the application. Our code is hosted on Github under the Mozilla Public License and is available for collaboration at https://github.com/iupuisoic/owa-copd.
The application use-cases were documented and initially the application was built as a single patient search and used to display the severity category of the patient, as a widget within the patient dashboard. The widget would always be shown for all patients and could not be customized or hidden for patients that did not have related observations or concepts. Based on the feedback of a clinician in our research group, we realized that this not only wasted valuable screen space, but also acted as a distraction to the clinician, who was attempting to decide on the diagnosis. On further analysis, we observed that several research articles on CPGs, pointed that the value of CPGs could be extended to a summary view of the patients under care. There are very limited implementations of CPGs in the documented literature as an overview tool [14]. Most CPGs, if ever implemented from paper to EHR systems, are usually part of Computerized Physician Order Entry (CPOE) where they act as decision support, to determine ordering of drugs or procedures [15], [16]. Finally, we implemented the CPG, such that, it can identify all the patients under observation of a clinical user (nurse, physician etc.) for COPD needs and classify them on severity, as well as create recommended orders for them in a single click.

In terms of the logic of implementing the CPG, the first check is for the concept of FEV1 or forced exhaled volume over 1 second. This percentage is essentially vital to the diagnosis of COPD, as per the clinical practice guidelines, as it determines the severity of the disease. The definition as well as normal, high, and low values were added to the concept dictionary, which are used for classifying patients. While oximetry levels were already included in the dictionary, the partial pressure of oxygen in arterial blood was not included. The definition of $\mathrm{PaO} 2$ was added as well as values to accommodate the newly added vital. Therefore, the definition as well as values were also added to the dictionary. The last concept to be used in the late stages of the project was the FEV1/FVC ratio. This ratio was vital to categorization of the patient's status and treatment.

With the concepts completed and placed in the dictionary, the task of creating a simulated COPD patient to test was next. Now that all definitions were added, the clinical findings for patient had to be tested for accurate classification. A simulated patient was created to begin to assess the function of the clinical practice guidelines. The patient, John, was created and vital signs were added. Unfortunately, due to the nature of the setup of OpenMRS only way to add vitals, observations, or encounters to a patient record that is not included on the forms is to manually create an observation.

In order to assess a patient and diagnose COPD, abnormal vitals and observations had to be included. Using these vitals included, the patient could now be labeled as a COPD patient based on the guidelines.

The final part of the project was to measure the accuracy and speed with which the application could classify patients on severity - at risk, mild, moderate, severe and very severe. We tested performance of the application, with a database which contained 758 patients, 3100 encounters and over 153,621 observations. These are de-identified patients, with dummy names that are part of the training dataset at our institution. This dataset has very limited patients related to COPD cases, as it is from CHCs with a focus on primary care. 


\section{RESULTS}

According to the application logic flow, all patients with chronic symptoms like coughing, shortness of breath, etc. and exposure to risk factors like smoking, or smoking family members, family history of COPD etc. were used to classify the patient at level 0 . For these patients, on the click of a button, the provider can order influenza vaccine, for their next scheduled appointment or during the present visit. This allows for planning resources required at the health facility. When patient's FEV1/FVC ration is less than $70 \%$ and FEV1 >= $80 \%$ the patient is classified as mild and for such patients the provider can order short-acting bronchodilator on the click of a button. For category 3 of moderate COPD, one or more longacting bronchodilators can be ordered. This is shown as an option box from which the provider can select the drugs that they want to order. This flexibility was determined to be important for providers who look at the patient demographic, family history, and other clinical factors that the CPG or EHR decision support cannot implement. Similarly, for patients classified Severe/Very Severe can order glucocorticosteroids, or even referral for surgeries. The logic for the referral was not built, since higher priority was given providers choice and we did not want to preempt or affect clinical decisions.

The result of this project is shown in Figure 1 below. After going through all CPGs designed in this project, every patient will be put into a position in that table and it will give the severity and treatment recommendations for the patients. You can see that we tested the four patients that are classified and the ordering that was done for the patients. The speed of the application in the classification was nearly instantaneous, just as would be any page to display on the web browser. All patients are searched for COPD guidelines observations and then those patients are run through the classification logic of the CPG.

\section{DISCUSSION}

The VA/DoD CPG provided the most clarity for us, which is likely a very subjective reading of the guidelines. We specifically looked for detailed flowcharts in the CPGs that would help it to be implemented in an EHR system. Some CPGs were clearly designed with human articulation in mind and were closely related to standard operating procedure.

Based on this project there is more work to be completed that can contribute to the effectiveness of clinical guideline use in an EMR system. Providers care for many patients at the same time. By the time a COPD patient revisit the provider with his result, 6 months have already passed since their last visit. Therefore, the provider may not remember every detail about this patient's condition to be able to make the correct diagnosis. This system we designed can help physicians put patients in the correct diagnostic spot by listing the CPG together with the patient's EHR. Physicians don't need to look at more than one place to collect all the needed information. As with all health-related issues, diagnosis is often dependent on clinical manifestations and testing which can be delayed, misinterpreted, or incorrect. The problem with such issues in healthcare is the number of patients that are untreated due to the non-diagnosing of chronic health issues. This is problematic for the use of CPG's in an EMR system as the patient must have a confirmed diagnosis of COPD in order for the guidelines to apply. This process has the potential to exclude several respiratory patients who have not been officially diagnosed with COPD. Furthermore, this could cause a delay and aggressive treatment and management based on the guidelines. Another concern that may arise with the use

Figure 1. An example of 4 patients classified based on the CPG of COPD

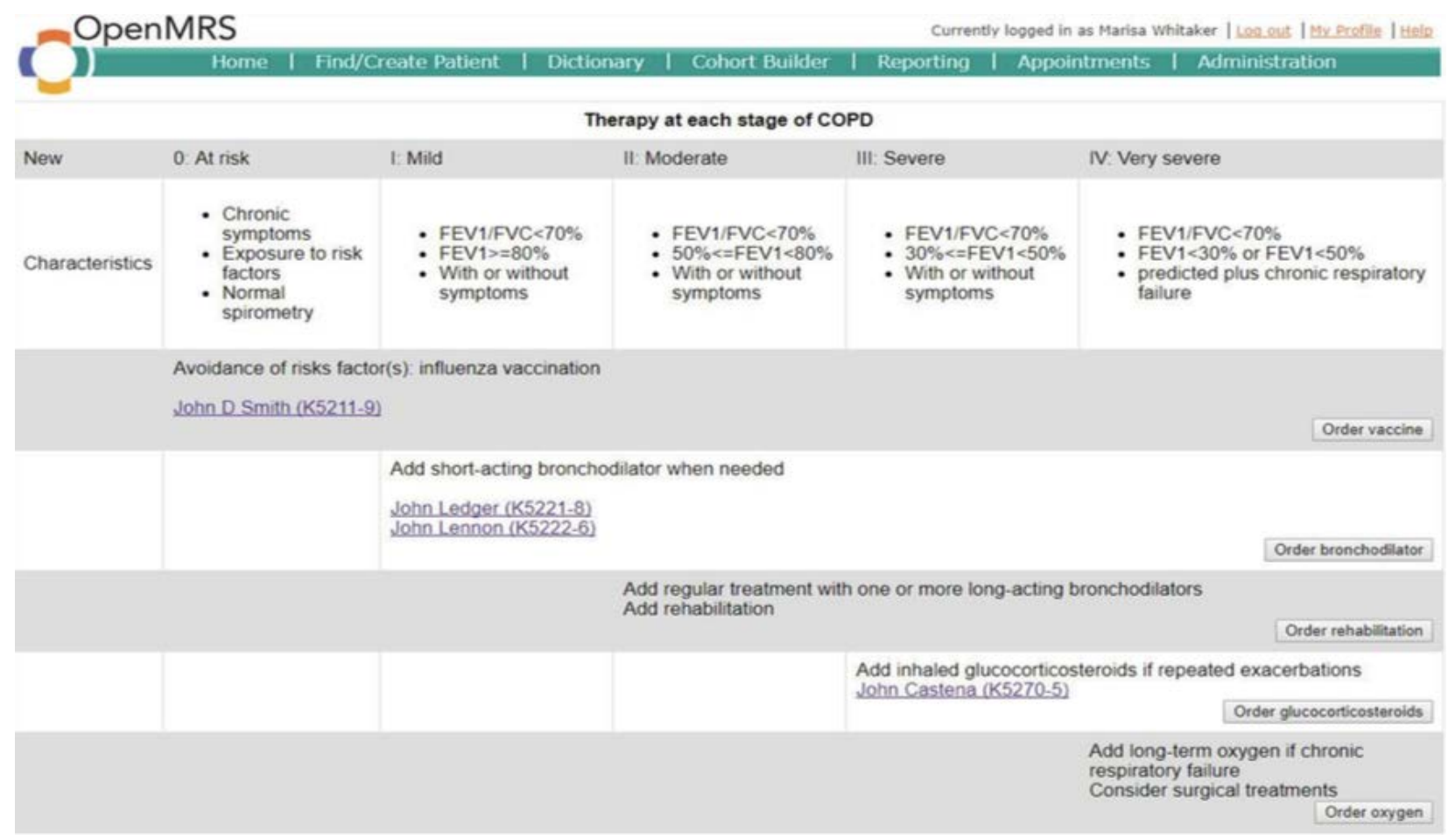


of the app is the universality of the application to other chronic disease. The symptoms and classifications may change for each disease and may require a more in-depth measurement of diagnosis and treatment based on guidelines.

Although there is more work to be done, the concepts involved in this application can be used in a multitude of settings including inpatient/outpatient, ED, and primary care. This project mainly focused on the diagnosis and treatment of COPD. We have planned follow up studies by implementing this in clinic setting and get feedback from clinicians and nurses. Although potential cost and time could be an issue in developing these project, the beneficial care provided to patients can be easily viewed and quantified using this application.

\section{CONCLUSION}

This paper demonstrates, what we believe is an innovative use of CPG in an EHR system. A new approach to provide a summary view of all patients under the provider's care for COPD. The use of OWA standard, such that it can then be ported to other EHR systems that can be used over web browsers. We demonstrate that the application logic for clinical practice guidelines can be implemented in a way that once data is available to it as a JSON, can be worked upon and displayed in a user-friend user interface. To create more efficient and effective care of chronic disease like COPD, these types of applications are becoming more relevant in the realm of medicine today. As providers are overwhelmed with the amount of information that is published and created each day, clinical practice guidelines are a way of creating a more informed decision-making process as patients are treated.

\section{REFERENCES}

[1] M. C. Politi, K. Y. Wolin, and F. Légaré, “Implementing Clinical Practice Guidelines About Health Promotion and Disease Prevention Through Shared Decision Making,” J. Gen. Intern. Med., vol. 28, no. 6, pp. 838-844, Jun. 2013.

[2] M. M. Cloutier, C. B. Hall, D. B. Wakefield, and H. Bailit, "Use of asthma guidelines by primary care providers to reduce hospitalizations and emergency department visits in poor, minority, urban children,” J. Pediatr., vol. 146, no. 5, pp. 591-597, May 2005.

[3] A. J. Guarascio, S. M. Ray, C. K. Finch, and T. H. Self, "The clinical and economic burden of chronic obstructive pulmonary disease in the USA,” Clin. Outcomes Res. CEOR, vol. 5, pp. 235-245, Jun. 2013

[4] S. H. Woolf, R. Grol, A. Hutchinson, M. Eccles, and J. Grimshaw, "Potential benefits, limitations, and harms of clinical guidelines," BMJ, vol. 318, no. 7182, pp. 527-530, Feb. 1999.

[5] E. R. Sutherland and R. M. Cherniack, "Management of Chronic Obstructive Pulmonary Disease,” N. Engl. J. Med., vol. 350, no. 26, pp. 2689-2697, Jun. 2004.

[6] "Management of Chronic Obstructive Pulmonary Disease (COPD) (2014) - VA/DoD Clinical Practice Guidelines.” [Online]. Available: https://www.healthquality.va.gov/guidelines/CD/copd/. [Accessed: 01-Sep-2017].

[7] K. Kunjan, T. Toscos, A. Turkcan, and B. N. Doebbeling, “A Multidimensional Data Warehouse for Community Health Centers," AMIA. Annu. Symp. Proc., vol. 2015, pp. 1976-1984, Nov. 2015.

[8] S. Purkayastha, J. W. Gichoya, and S. A. Addepally, "Implementation of a single sign-on system between practice, research and learning systems,” Appl. Clin. Inform., vol. 8, no. 1, pp. 306-312, 2017.

[9] J. Jaffe, "Web at 25, W3C at 20: An Opportunity to Reflect and Look to the Future," IEEE Internet Comput., vol. 18, no. 4, pp. 74-78, Jul. 2014.
[10] Y.-S. Chang, S.-H. Lee, J.-C. Kim, and Y.-J. Lim, "Study on mobile mashup webapp development tools for different devices and user groups," in The International Conference on Information Networking 2014 (ICOIN2014), 2014, pp. 433-438.

[11] D. E. Robbins, A. Grüneberg, H. F. Deus, M. M. Tanik, and J. Almeida, "TCGA Toolbox: An Open Web App Framework for Distributing Big Data Analysis Pipelines for Cancer Genomics," in Proceedings of the International Conference on Bioinformatics, Computational Biology and Biomedical Informatics, New York, NY, USA, 2013, p. 62:62-62:67.

[12] K. D. Mandl et al., "The SMART Platform: early experience enabling substitutable applications for electronic health records," J. Am. Med. Inform. Assoc., vol. 19, no. 4, pp. 597-603, Jul. 2012.

[13] “generator-openmrs-owa," npm. [Online]. Available: https://www.npmjs.com/package/generator-openmrs-owa. [Accessed: 13-Sep-2017].

[14] D. Isern and A. Moreno, "Computer-based execution of clinical guidelines: a review,” Int. J. Med. Inf., vol. 77, no. 12, pp. 787-808, 2008.

[15] F. Sonnenberg and C. Hagerty, "Computer-interpretable clinical practice guidelines," Are We Are We Going, pp. 145-158, 2006.

[16] D. F. Sittig et al., "Grand challenges in clinical decision support," $J$. Biomed. Inform., vol. 41, no. 2, pp. 387-392, 2008. 